\title{
Antiviral Effect of Resveratrol in Piglets Infected with Virulent Pseudorabies Virus
}

\author{
Xinghong Zhao ${ }^{1,+, \ddagger}$, Wenzhi Tong ${ }^{1,+}{ }^{1}$, Xu Song ${ }^{1,+}$, Renyong Jia ${ }^{2, *}$, Lixia Li ${ }^{1}$, Yuanfeng Zou ${ }^{1}$ (D), \\ Changliang He ${ }^{1}$, Xiaoxia Liang ${ }^{1}$, Cheng Lv ${ }^{1}$, Bo Jing ${ }^{1}$, Juchun Lin ${ }^{1}$, Lizi Yin ${ }^{1}$, Gang Ye ${ }^{1}$, \\ Guizhou Yue ${ }^{3}$, Yin Wang ${ }^{2}$ and Zhongqiong Yin ${ }^{1, *}$ \\ 1 Natural Medicine Research Center, College of Veterinary Medicine, Sichuan Agricultural University, \\ Chengdu 611130, China; zhaoxinghong8@163.com (X.Z.); 13056679601@163.com (W.T.); \\ songx@sicau.edu.cn (X.S.); lilixia905@163.com (L.L.); yuanfengzou@sicau.edu.cn (Y.Z.); \\ lorri190@126.com (C.H.); liangxiaoxia@sicau.edu.cn (X.L.); lvcheng1980@163.com (C.L.); \\ jingbooo@163.com (B.J.); juchunlin@126.com (J.L.); yinlizi@hotmail.com (L.Y.); yegang800206@163.com (G.Y.) \\ 2 Key laboratory of Animal Disease and Human Health of Sichuan Province, Sichuan Agricultural University, \\ Chengdu 611130, China; yaanwangyin@tom.com \\ 3 College of Science, Sichuan Agricultural University, Ya'an 625014, China; yueguizhou@sicau.edu.cn \\ * Correspondence: cqrc_jry@163.com (R.J.); yinzhongq@163.com (Z.Y.) \\ + These authors contribute equally to this work. \\ $\ddagger$ Present address: Department of Molecular Genetics, Faculty of Science and Engineering, Nijenborgh 7, \\ 9747 AG, University of Groningen, 9713 GZ Groningen, The Netherlands
}

Received: 15 July 2018; Accepted: 23 August 2018; Published: 27 August 2018

\begin{abstract}
Pseudorabies virus (PRV) is one of the most important pathogens of swine, resulting in devastating disease and economic losses worldwide. Nevertheless, there are currently no antiviral drugs available for PRV infection. Resveratrol (Res) was identified to exert its antiviral activity by inhibiting the PRV replication in preliminary investigations. In our previous study, we found that Res has anti-PRV activity in vitro. Here, we show that Res can effectively reduce the mortality and increase the growth performance of PRV-infected piglets. After Res treatment, the viral loads significantly $(p<0.001)$ decreased. Pathological symptoms, particularly inflammation in the brain caused by PRV infection, were significantly $(p<0.001)$ relieved by the effects of Res. In Res-treated groups, higher levels of cytokines in serum, including interferon gama, interleukin 12, tumor necrosis factor-alpha and interferon alpha were observed at 7 days post infection. These results indicated that Res possesses potent inhibitory activity against PRV-infection through inhibiting viral reproduction, alleviating PRV-induced inflammation and enhancing animal immunity, suggesting that Res is expected to be a new alternative control measure for PRV infection.
\end{abstract}

Keywords: antiviral activity; resveratrol; virulent pseudorabies virus; piglets

\section{Introduction}

Pseudorabies virus (PRV; also called Aujeszky's disease virus or suid herpesvirus type 1), is a member of the Alphaherpesvirinae subfamily within the family Herpesviridae, and is the causative agent of Aujeszky's disease (AD), which is one of the most devastating infectious diseases of swine and results in significant economic losses for the swine industry [1,2]. AD is a contagious disease which is characterized by encephalomyelitis, frequently accompanied by inflammation of the upper respiratory tract and lungs [3]. In general, PRV mainly infects pigs at various production phases, such as causing nervous system disorders and high mortality in newborn piglets, respiratory disorders in older pigs, and reproductive failure in sows $[1,4,5]$. Despite widespread use of the Bartha-K61 vaccine in controlling PRV, AD continues to be one of the most important diseases in pigs in many 
countries, particularly in regions with dense pig populations, including China [6,7]. Outbreaks of AD in pigs caused by PRV variants happen frequently, even in herds immunized with the Bartha-K61 vaccine. Moreover, new prevalent PRV strains have caused great economic losses to the swine industry in China since 2011 [5,8-13].

Resveratrol (3,5,4-trihydroxystilbene, Res), a non-flavonoid polyphenol compound exists widely in several higher plants. Res has been reported to have antiviral activity against a series of viruses either in vitro or in vivo, including herpesviruses [14-16], retroviruses [17,18], respiratory syncytial virus [19] and human immunodeficiency virus type 1 [20]. Although Res has been known to have antiviral activity for many years, the use of Res to treat virus infection in relevant virus-host systems has rarely been undertaken.

Previously, we determined the anti-PRV activity of Res for the first time in vitro. The results showed that Res could effectively inhibit virulent PRV replication in vitro [21]. However, little is known about in vivo antiviral activity of Res against PRV. In this study, the anti-PRV activity of Res in piglets infected with virulent PRV was determined in order to develop a new alternative control measure for PRV infection and investigate the antiviral activity of Res in a relevant virus-host system.

\section{Materials and Methods}

\subsection{Compounds}

Resveratrol (Purity of 98\%; Sigma, St. Louis, MO, USA) was dissolved in $0.5 \%$ carboxymethylcellulosephosphate-buffered saline (SCMC-PBS) before use.

\subsection{Virus and Piglets}

Virulent PRV (Rong A strain, purchased from China Veterinary Culture Collection Center) was propagated in PK-15 cells. 28-day-old healthy (before the Res administration, the piglets had been investigated for 7 days, with no disease symptoms being observed) piglets were purchased from a remote mountain village (Leshan, China), and no PRV gB-specific antibody were detected through ELISA assay (IDEXX, Westbrook, ME, USA). Piglets were maintained under normal daylight and fed with a standard commercial diet and water ad libitum.

\subsection{Ethics Statement}

All procedures involving animals and their care in this study were approved by the Ethics Committee of Sichuan Agricultural University according to the Regulation of Experimental Animal Management (State Scientific and Technological Commission of the People's Republic of China, No.2, 1988) and the Interim Measures of Sichuan Province Experimental Animal Management (Science and Technology Bureau of Sichuan, Sichuan, China, No.25, 2013) (the approved permit number is XF2014-18).

\subsection{Experimental Design}

Fifty 35-day-old piglets were randomly divided into five groups. Before infection, the piglets in the Res-treated groups were administered Res through addition into the commercial diet at doses of 30 (Res-H), 10 (Res-M) and 3 (Res-L) mg/kg body weight daily for 7 days. The piglets in the untreated and non-infected groups received only the commercial diet. At 42-days-old, piglets were infected intranasally with $1 \mathrm{~mL}$ of $2 \times 10^{6} \mathrm{TCID}_{50}$ PRV, except the non-infected group. After infection, the piglets in all of the groups were fed the standard commercial diet. The infected piglets were immediately administered Res solutions orally at doses of 90 (Res-H), 30 (Res-M) and 10 (Res-L) mg/kg body weight twice daily for 21 days, respectively. The dosages of Res before and after infection were based on our previous research: Fu et al., 2018 and Zhao et al., 2017, respectively [21,22]. The piglets in the untreated and non-infected groups were given the same volume of SCMC-PBS. All animals were physically examined daily, and nasal swabs were taken at regular intervals after infection to monitor 
virus excretion. Serum samples were taken at $0,7,14$, and 21 days post infection (dpi). Three randomly selected piglets were subjected to necropsy in each group at 7 and $21 \mathrm{dpi}$. The rearing conditions were based on the Guidelines of the International Committee on Laboratory Animals.

\subsection{Analysis of Viral Load by Real-Time PCR}

The PRV load of piglets was monitored by the real-time fluorescence quantification PCR (FQ-PCR) of nasal swab and brain tissue. The total DNA was isolated from the nasal swabs and brains tissue by TIANamp Swab DNA Kit (Tiangen Biotech, Beijing, China) and Genomic DNA Extraction Kit (TaKaRa, Tokyo, Japan), respectively. The upstream and downstream primers were $5^{\prime}$ - ACAAGTTCAAGGCCC ACATCTAC $-3^{\prime}$ and $5^{\prime}$ - GTCYGTGAAGCGGTTCGTG AT $-3^{\prime}$, respectively, which were used to amplify a 95-bp fragment of the glycoprotein B gene of PRV (GenBank accession no. KJ526438). A 17-bp probe (5'- ACGTCATCGTCACGACC - $3^{\prime}$ ) complementary to an internal region between two primers was selected and labelled with carboxyfluorescein at the $5^{\prime}$ end and with carboxytetramethylrhodamine at the $-3^{\prime}$ end. The FQ-PCR was analyzed by using SsoAdvancedTM Universal Probes Supermix (BIO-RAD, Hercules, CA, USA) with a Bio-Rad CFX96TM Manager software system according the method described in our previous study [21].

\subsection{Histopathological Analysis}

Histopathological lesions of PRV-infected piglets treated with or without Res were investigated. Heart, liver, kidney, lung, spleen and brain tissues were procured at $7 \mathrm{dpi}$, preserved in $4 \%$ paraformaldehyde, and enclosed in paraffin for subsequent histopathological examination. A $5 \mu \mathrm{m}$ section of each organ tissue was stained with hematoxylin and eosin. Each section was analyzed under an optical microscope (Nikon eclipse 80i, Tokyo, Japan). Three slides from different parts of each tissue ( 3 piglets per group) were analyzed. The whole lesions for each tissue were scored by multiplying the degree of severity $(0=$ no lesions, $1=$ mild lesions, $2=$ moderate lesions, and $3=$ severe lesions $)$ by the extent of lesions $(1=$ low extent, 2 = intermediate extent, and $3=$ large extent $)$ [23].

\subsection{Serum Cytokines Assay}

The concentrations of cytokines in serum, including interferon alpha (IFN- $\alpha$ ), interferon gama (IFN- $\gamma)$, tumor necrosis factor-alpha (TNF- $\alpha$ ) and interleukin 12 (IL-12), were detected by using an ELISA kit according to the manufacturer's instructions (Shanghai Enzyme-Linked Biotechnology Co., Ltd., Shanghai, China).

\subsection{Statistical Analysis}

Data was expressed as the mean \pm S.D and the statistical significance of the data was assessed using a two-tailed Student's $t$-test with GraphPad Prism software 5 (LaJolla, CA, USA). Correlation analyses were evaluated by Pearson r2, ns: $p>0.05,{ }^{*} p<0.05$ and $p<0.001$.

\section{Results}

\subsection{Resveratrol Reduced Mortality and Increased Body Weight Gained by Piglets Infected with Virulent PRV}

As shown in Table 1, there were no deaths among groups before $6 \mathrm{dpi}$, but the piglets began to die in the untreated group at $6 \mathrm{dpi}$; in contrast, there were no deaths in the Res-treated groups at the same time. The groups treated with Res exhibited a high protection rate $(100 \%$, in Res-H and Res-M groups) against PRV infection. However, only six out of ten animals survived in the untreated group. 
Table 1. Resveratrol treatment reduced the mortality of PRV-infected piglets.

\begin{tabular}{cccccc}
\hline \multirow{2}{*}{ Days Post Infection } & \multicolumn{5}{c}{ Survival Rate (\%) } \\
\cline { 2 - 6 } & Res-H & Res-M & Res-L & Untreated & Non-Infected \\
\hline 1 & 100 & 100 & 100 & 100 & 100 \\
2 & 100 & 100 & 100 & 100 & 100 \\
3 & 100 & 100 & 100 & 100 & 100 \\
4 & 100 & 100 & 100 & 100 & 100 \\
5 & 100 & 100 & 100 & 100 & 100 \\
6 & 100 & 100 & 100 & 80 & 100 \\
$7^{\text {a }}$ & 100 & 100 & 90 & 60 & 100 \\
\hline
\end{tabular}

Survival rates of the PRV-infected piglets treated with resveratrol (RV-H, RV-M, RV-L, respectively) and untreated were recorded at $7 \mathrm{dpi}\left(n=10\right.$, in each group). ${ }^{\text {a }}$ Date of last death.

Changes in body weight were analyzed over 21 dpi (Figure 1). Compared with the non-infected group, all infected piglets had a reduced gain in body weight. However, compared with the untreated group, the body weight gained increased in the Res-treated groups in a dose-dependent manner.

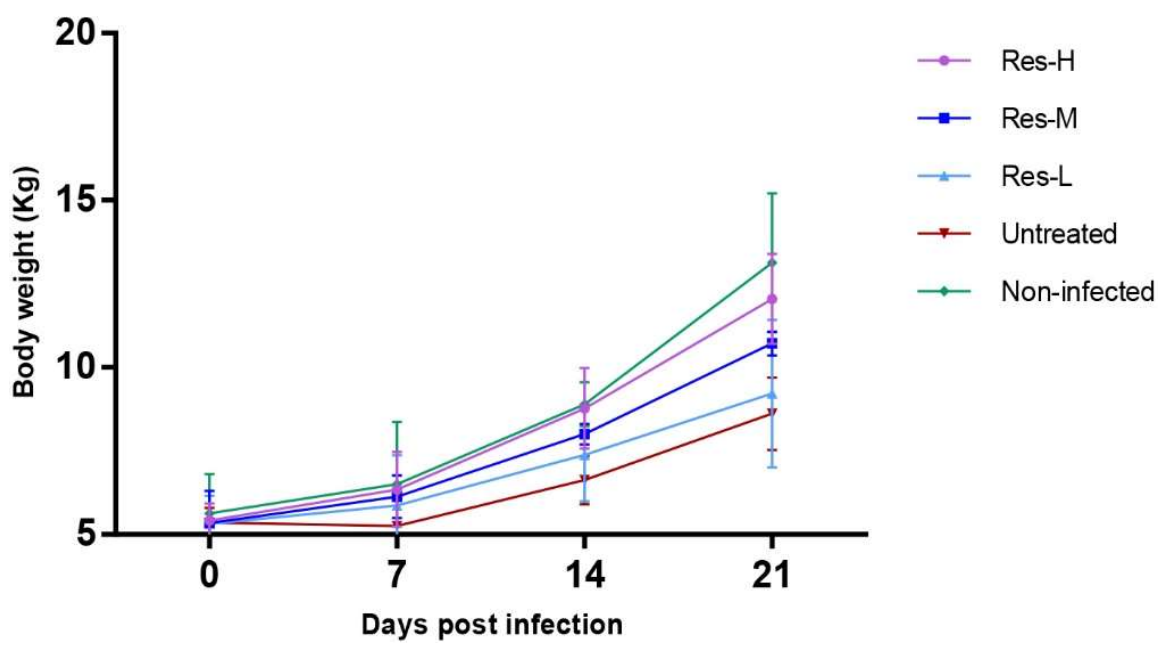

Figure 1. Body weight changes following PRV infection.

\subsection{The Viral Load of Nasal Swab and Brain Were Depressed by Res}

The nasal swabs of each group were collected at $0,3,5,7,10,14$ and $21 \mathrm{dpi}$, and the viral copies were assayed by FQ-PCR. The results are shown in Figure 2. In the untreated group, virus excretion began to increase rapidly at $3 \mathrm{dpi}$, while lower viral loads were detected in the Res-treated groups. The viral loads in the Res-treated groups were significantly $(p<0.001)$ lower than that in the untreated group. At $5 \mathrm{dpi}$, viral loads increased in all infected groups. The Res-treated groups had significantly $(p<0.001)$ lower viral loads compared to the untreated group. At $7 \mathrm{dpi}$, viral loads in all infected groups decreased, and the viral loads in the Res-treated groups were significantly $(p<0.001)$ lower than those in the untreated group. At $10 \mathrm{dpi}$, viral loads in Res-H and Res-M groups continually decreased, while the viral loads in Res-L and untreated groups increased; the viral loads in the Res-treated groups were significantly $(p<0.001)$ lower than those in the untreated group. At $14 \mathrm{dpi}$, there was no PRV genome detected among the groups, except one piglet in the untreated group. There was no PRV genome detected at $21 \mathrm{dpi}$ among any of the groups. 


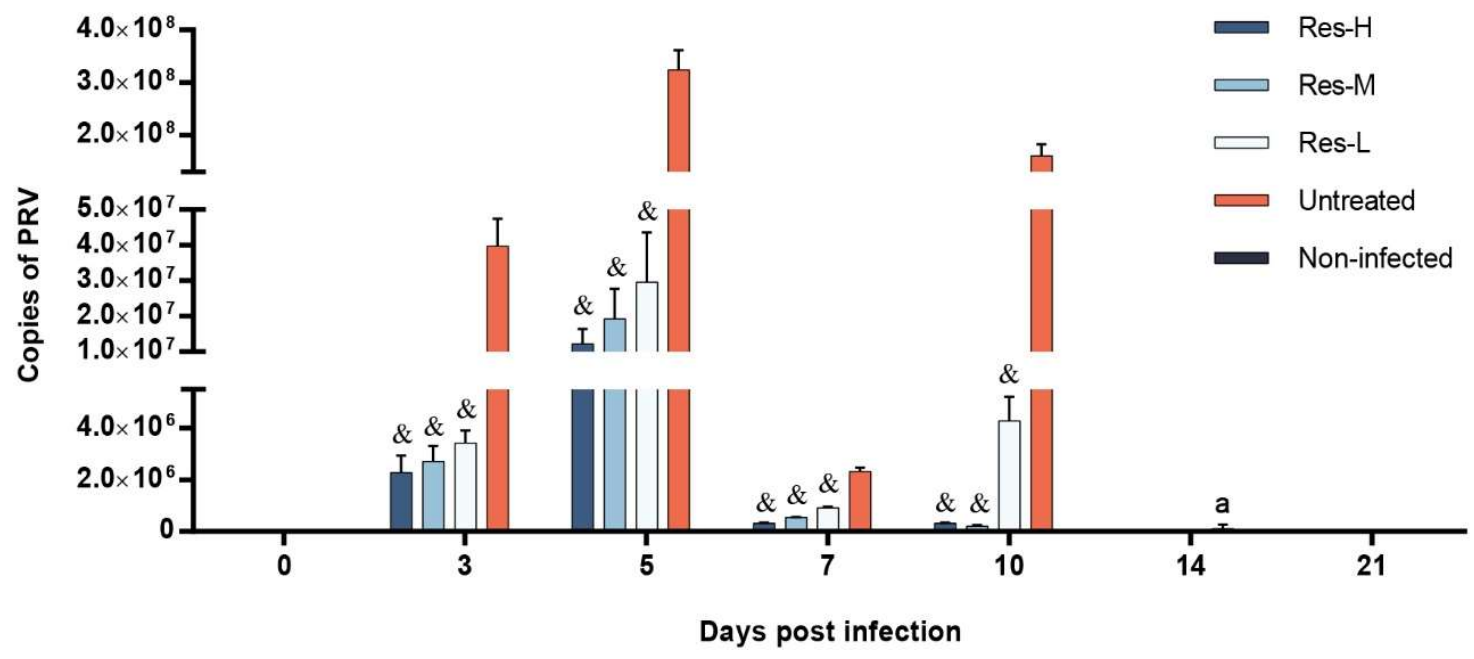

Figure 2. Copies of the PRV genome per nasal swab sample of piglets. Copies of PRV genome in nasal of the piglets were analyzed by FQ-PCR at $0,3,5,7,10,14$ and 21 dpi (at every selected time point, all of the living piglets were tested, $n \geq 3$, in each group). There was no PRV genome detected in the non-infected group at any time point and no PRV genome detected at 0, 14 and $21 \mathrm{dpi}$ among the groups. ${ }^{a}$ There was one piglet detected with PRV in the untreated group at $14 \mathrm{dpi}$. Correlation analyses were evaluated by Pearson $\mathrm{r} 2, \& p<0.001$ vs. untreated group.

The brains of each group (three piglets per group) were collected at 7 and $21 \mathrm{dpi}$, and the viral copies were assayed by FQ-PCR. The results are shown in Figure 3. In the untreated group, viral copies were significantly $(p<0.001)$ higher than those in the Res-treated groups at $7 \mathrm{dpi}$. There was no PRV genome detected at $21 \mathrm{dpi}$ among any of the groups.

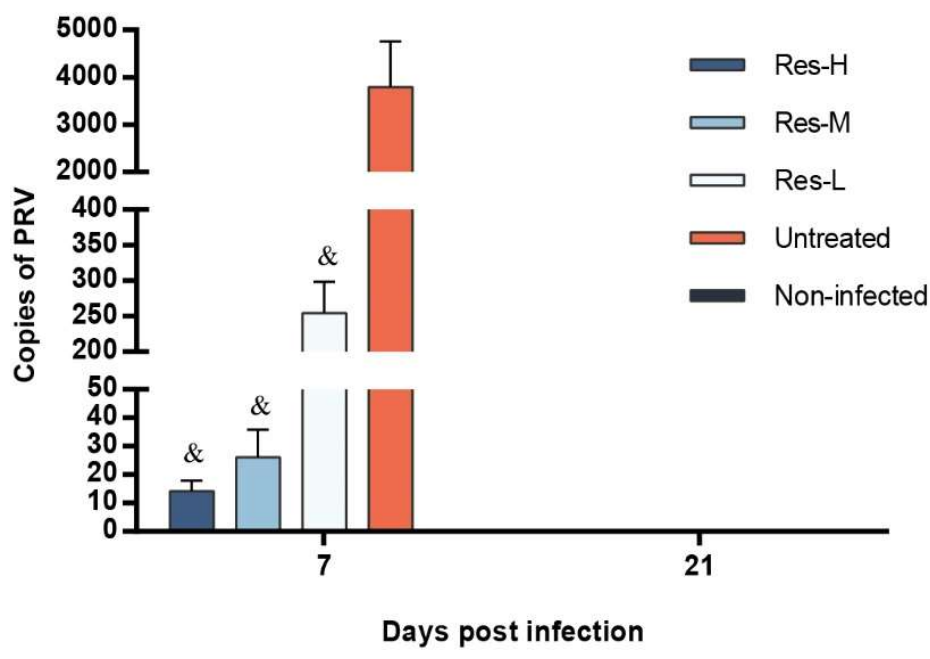

Figure 3. Copies of PRV genome per microgram brain of piglets. Copies of the PRV genome in the brain of the piglets were analyzed by FQ-PCR at 7 and $21 \mathrm{dpi}$ ( $n=3$ in each group). There was no PRV genome detected in the non-infected group at any time point and no PRV genome detected at $21 \mathrm{dpi}$ among the groups. Correlation analyses were evaluated by Pearson $\mathrm{r} 2, \& p<0.001$ vs. untreated group.

\subsection{Res Reduced the Pathological Lesions of PRV-Infected Piglets}

The PRV-infected piglets of each group (three piglets per group) were dissected at $7 \mathrm{dpi}$. The different histopathological lesions of brain, liver, lung, kidney, spleen and heart of PRV-infected piglets in the untreated group and Res-M-treated groups are shown in Figure 4. In the brains of the untreated group, a large number of lymphocytes surround the blood vessel, showing tubular 
infiltration ( $\uparrow$, Figure 4B). There were fewer lymphocytes observed in the brain of the Res-M-treated group (Figure 4A). In the lungs of the untreated group, severe pulmonary abscessations were observed; the alveolar structure disappeared and was infiltrated with many neutrophilic granulocytes ( $\uparrow$, Figure 4E). There were mild lesions observed in the lungs of the Res-M-treated group (Figure 4D): thickened alveolar wall and infiltration with some of the neutrophilic granulocytes. In the kidneys of PRV-infected piglets (Figure 4H), severe capillary hyperemia $(\uparrow)$ was observed. The normal histological structure of the kidney was observed in the Res-M-treated group (Figure 4G). In the livers of the untreated group (Figure 4K), focal necrosis with lymphocytes infiltrated was observed ( $\uparrow$, Figure $4 \mathrm{~K}$ ). Granular degeneration of hepatocytes was observed in the Res-M-treated group (Figure 4J). However, compared with the untreated group, no focal necrosis was observed (Figure 4J). In the spleens of the untreated group (Figure $4 \mathrm{~N}$ ), splenic corpuscles were demolished and had disappeared, red pulp was widened, and white pulp was atrophied $(\uparrow)$. The normal histological structure of spleen was observed in the Res-M-treated group (Figure 4M). Vacuolar degeneration appeared in the hearts of the untreated group $(\uparrow$, Figure 4Q), while in the Res-M-treated group, the heart basically kept normal histological structure (Figure 4P). These observations were also proven by the lesional score (Table 2).

Table 2. Lesional scores for each group at $7 \mathrm{dpi}^{\mathrm{a}}$.

\begin{tabular}{ccccc}
\hline Group & Res-H & Res-M & Res-L & Untreated \\
\hline Brain & $0.72 \pm 0.15^{\&}$ & $0.69 \pm 0.21^{*}$ & $3.90 \pm 0.36^{*}$ & $8.02 \pm 0.85$ \\
Lung & $3.54 \pm 0.06^{*}$ & $3.73 \pm 0.21^{*}$ & $5.74 \pm 0.43$ & $7.41 \pm 1.43$ \\
Kidney & $0.65 \pm 0.06^{*}$ & $0.99 \pm 0.10^{*}$ & $4.32 \pm 0.31 *$ & $6.39 \pm 0.55$ \\
Liver & $1.84 \pm 0.24 *$ & $2.01 \pm 0.30^{*}$ & $4.75 \pm 0.23$ & $6.33 \pm 1.13$ \\
Spleen & N.D. & N.D. & $2.28 \pm 0.42^{*}$ & $5.38 \pm 0.86$ \\
Heart & $0.88 \pm 0.79 *$ & $0.77 \pm 1.33 *$ & $2.48 \pm 0.46^{*}$ & $5.33 \pm 0.66$
\end{tabular}

a Lesional scores of each organ were obtained by multiplying the degree of severity $(0=$ no lesions, $1=$ mild lesions, $2=$ moderate lesions, and 3 = severe lesions) with the extent of lesions $(1=$ low extent, $2=$ intermediate extent, and $3=$ large extent). N.D. means no lesions detected. The values are presented as means \pm standard deviation $\left(n=3\right.$, in each group). Correlation analyses were evaluated by Pearson r2, ns: $p>0.05,{ }^{*} p<0.05$ and ${ }^{\&} p<0.001$ vs. untreated group.

\subsection{The Concentrations of Cytokines Were Affected by Res}

ELISA assays were used to detect the concentrations of IFN- $\alpha$, IFN- $\gamma$, TFN- $\alpha$ and IL-12 in the serum which was separated from the venous blood at $0,7,14$ and 21 dpi. The results are shown in Figure 5. At 0 dpi, the concentrations of IL-12, TNF- $\alpha$, IFN- $\alpha$ and IFN- $\gamma$ showed no significant difference among groups. At $7 \mathrm{dpi}$, compared with the non-infected group, the concentrations of IL-12 and IFN- $\alpha$ were significantly $(p<0.001)$ decreased in the untreated group, while the decreasing tendency was significantly $(p<0.05$ or 0.001 ) inhibited by Res treatment in a dose-dependent manner. Surprisingly, the concentrations of IFN- $\gamma$ in the untreated group were decreased compared to the non-infected group; however, compared with the non-infected group, the concentration of IFN- $\gamma$ in Res-treated groups were significantly $(p<0.05$ or 0.001$)$ increased due to the Res treatment. The concentration of TFN- $\alpha$ was significantly $(p<0.001)$ increased by the infection of PRV. Moreover, compared with the untreated group, Res significantly $(p<0.05$ or 0.001$)$ increased the concentration of TFN- $\alpha$. At $14 \mathrm{dpi}$, compared to the non-infected group, the concentrations of IL-12 and TFN- $\alpha$ were decreased in the untreated group, while the decreasing tendency was significantly $(p<0.05$ or $0.001)$ inhibited by Res treatment. The concentration of IFN- $\gamma$ was significantly $(p<0.001)$ increased due to PRV-infection. Moreover, the concentrations of IFN- $\gamma$ in Res-treated groups were significantly $(p<0.001)$ higher compared to the untreated group. The concentration of IFN- $\alpha$ showed no significant difference among the groups. At $21 \mathrm{dpi}$, there were no significant differences of IL-12, TNF- $\alpha$, IFN- $\alpha$ and IFN- $\gamma$ levels among the groups. 



Liver

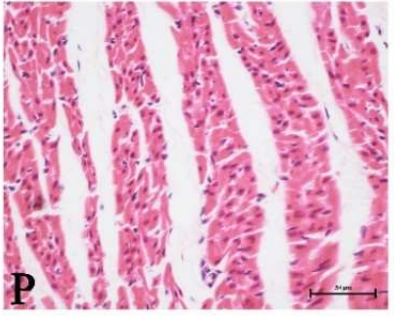

Res-M

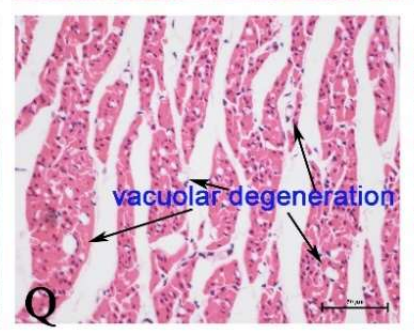

Untreated

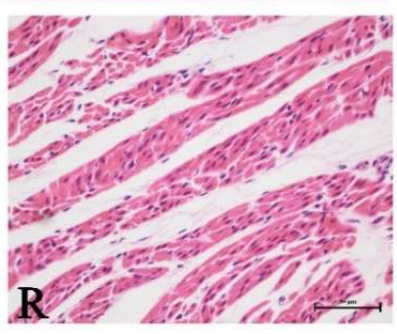

Non-infected

Figure 4. Effect of Res on the microstructures of tissues of piglets at 7 dpi. Panels (A-C): brain of Res-M, Untreated and Non-infected groups, respectively. Panels (D-F): lung of Res-M, Untreated and Non-infected groups, respectively. Panels (G-I): kidney of Res-M, Untreated and Non-infected groups, respectively. Panels (J-L): liver of Res-M, Untreated and Non-infected groups, respectively. Panels (M-O): spleen of Res-M, Untreated and Non-infected groups, respectively. Panels (P-R): heart of Res-M, Untreated and Non-infected groups, respectively. Final magnification $\times 400$, bars $=100 \mu \mathrm{m}$. 

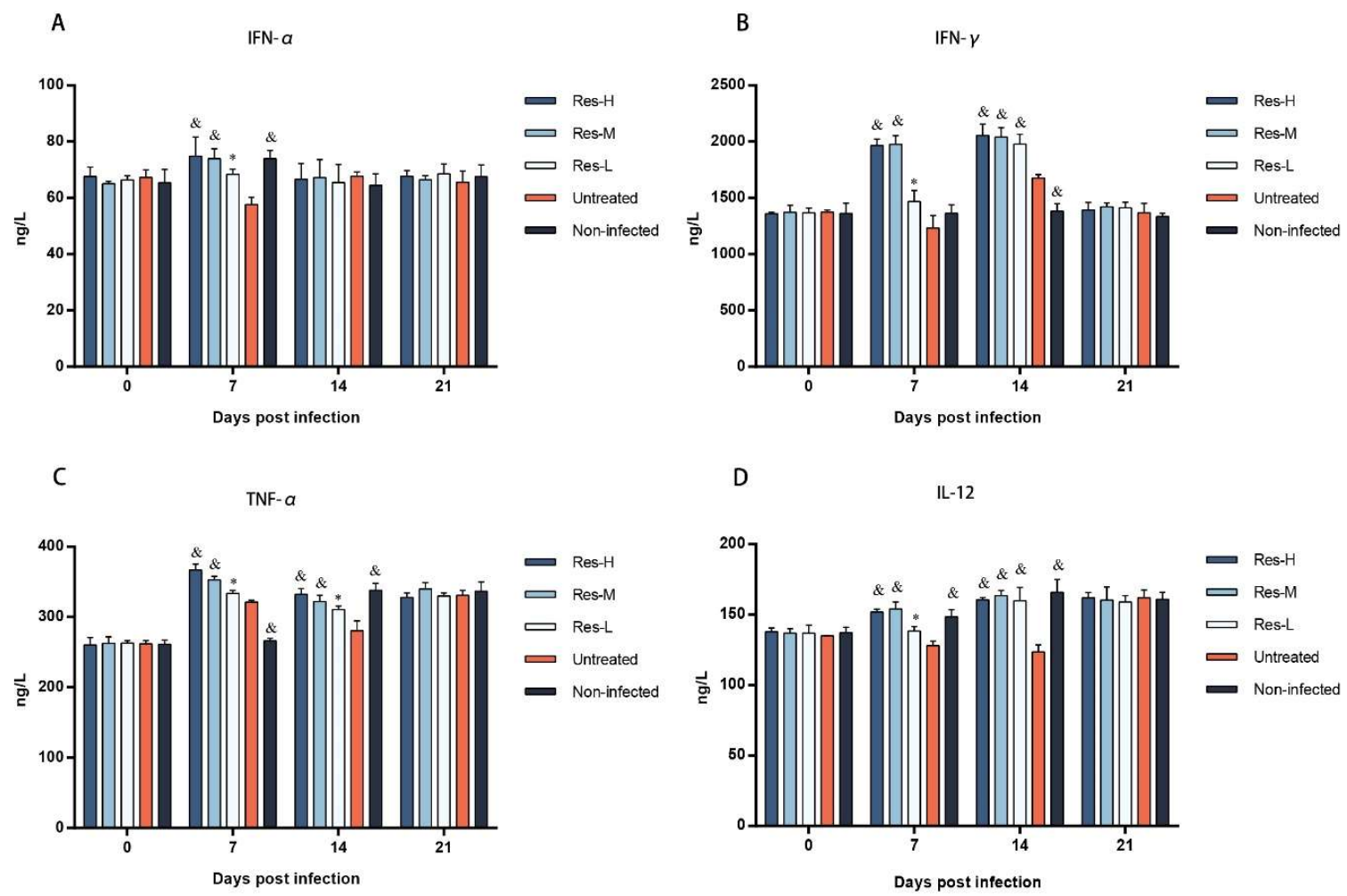

Figure 5. The concentrations of IFN- $\alpha$ (A), IFN- $\gamma(\mathbf{B})$, TNF- $\alpha($ C) and IL-12 (D) in serum obtained from test piglets. Four cytokines in the serum of the piglets were assayed at 0, 7, 14 and 21 dpi (at each selected time point, all of the living piglets were tested, $n \geq 3$ in each group). Correlation analyses were evaluated by Pearson r2, ns: $p>0.05,{ }^{*} p<0.05$ and ${ }^{\&} p<0.001$ vs. untreated group.

\section{Discussion}

Although Res has been known to have antiviral activity for many years, the use of Res to treat virus infection in a relevant virus-host system has rarely been done. In our previous study, Res showed potent antiviral activity against virulent duck enteritis virus [14,24]; we also found that Res possessed potent antiviral activity against PRV [21].

This study confirms that Res has a potent antiviral effect in PRV-infected piglets. Addition of Res could reduce mortality rate caused by PRV infection. No piglets died in the Res-H and Res-M groups, and nine out of ten piglets survived in the Res-L-treated group. It should be noted that the body weight gains of the Res-treated groups were higher than that in the untreated group in a dose-dependent manner. Our previous study showed that piglets (without infection) treated with Res were able to gain an insignificant amount of body weight more than control piglets (i.e., non-infected piglets) [22]. Here, we show that Res could help PRV-infected piglets to gain more body weight. These results indicate that Res could be used to reduce the economic losses in PRV-infected piglets by increasing their survival rate and growth performance. These results are consistent with our previous study [14].

Viral load is an important and direct parameter in the evaluation of antiviral effects in vivo $[14,23,25,26]$. The viral loads of brain tissue and nasal swabs were the most important parameters in the evaluation of virus proliferation and excretion in PRV-infected piglets, respectively $[25,26]$. In this study, the viral loads were detected by FQ-PCR. The results revealed that Res could significantly inhibit virus excretion, and efficiently reduce virus reproduction. The levels of viral copies in the brain were positively linked to the clinical parameters of infected piglets, which were confirmed by our previous study that Res exerts antiviral activities by inhibiting viral reproduction $[14,21,24]$. 
The antiviral effects of Res on PRV-infected piglets were also supported by histopathological observations. In this study, obvious lesions were detected in the brain, lung, kidney, liver, spleen and heart after infection (Figure $4 \mathrm{~B}, \mathrm{E}, \mathrm{H}, \mathrm{K}, \mathrm{N}, \mathrm{Q}$ ), which were consistent with the previous study [8]. Res significantly decreased the tissues lesions. The results indicated positive therapeutical effects of Res on tissue lesions caused by PRV-infection. Given the high survival ratio and growth performance in the Res-treated groups, we can conclude that Res could effectively inhibit PRV reproduction and suppress the inflammations induced by PRV-infection, and thus decrease the tissue lesions. These results were consistent with our previous reports, which showed that Res could suppress tissue lesions and inflammation $[14,27,28]$.

The immune system plays a key role in protecting the body from foreign pathogens through either innate immunity or acquired immunity. It is well established that innate factors, including IFN- $\alpha$, IFN- $\gamma$, TNF- $\alpha$ and IL-12, play a critical role in inhibiting virus infections; thus, the levels of these cytokines are critical for antiviral immunity [29-33]. In this study, the levels of cytokines (IFN- $\alpha$, IFN- $\gamma$, TNF- $\alpha$ and IL-12) were detected. The results show that the levels of TNF- $\alpha$ in Res-treated groups were significantly higher than that in the untreated group, and the depressed productions of IFN- $\alpha$, IFN- $\gamma$ and IL-12 induced by PRV-infection were significantly improved by Res treatment, especially the level of IFN- $\gamma$. These results were consistent with our previous reports, which showed that Res could increase the concentrations of IFN- $\gamma$ in the serum of piglets [22]. Smith et al. reported that IFN- $\gamma$-mediated mechanisms play a critical role in the control of and recovery from acute Herpesviridae virus infection [34]. Based on the combination of this information with our results, we can conclude that the higher levels of IFN- $\gamma$ in the Res-treated groups might be one of the primary reasons for Res having an antiviral effect against virus infection.

In conclusion, Res showed potent antiviral activity on PRV infection in piglets. It was able to decrease the mortality of PRV-infected piglets, enhance growth performance, inhibit viral reproduction, alleviate tissue inflammation and lesions, and improve the levels of cytokines in PRV-infected piglets. The antiviral activity of Res might mainly be attributed to the inhibitory effect on PRV proliferation and immunomodulatory effects of IFN- $\gamma$. Resveratrol exhibits potential for PRV control, and further studies should be conducted to evaluate the antiviral activity of Res against infection with other viruses in a relevant virus-host system.

Author Contributions: X.Z. and W.T. performed most experiments and analyzed the data. Y.Z., L.L., C.H., X.L., C.L., L.Y., G.Y. (Gang Ye), G.Y. (Guizhou Yue), B.J., J.L. and Y.W. helped with some experiments. R.J., Z.Y., and X.Z. conceived and designed the experiments, and Z.Y., X.S., and X.Z. wrote the manuscript.

Funding: This study was supported by the National Natural Science Foundation of China (Grant No. 31602110), the Sichuan Province Science and Technology Support Program (Grant No. 2017NZZJ036, 2018NZ0043 and 2018NZ0064), the Traditional Chinese Medicine Industry Innovation Team of Sichuan Province (Grant No. 2017C015), the Sichuan Veterinary Medicine and Drug Innovation Group of China Agricultural Research System (CARS-SVDIP), and the Agricultural Technology Research and Development Project of Chengdu (Grant No. 2015-NY02-00266-NC).

Acknowledgments: We thank Qiankun Cui, Ye Tian, Shufan Chen, Lu Bai, Qiuting Fu, Yi Yang, Kai Sun and Jiankang Yu for them experimental assistance. We thank Max Dalglish (UK), Adriaan Jaarsma (The Netherlands) and Koen Giesbers (The Netherlands) for checking the grammar and spelling.

Conflicts of Interest: The authors declare no conflict of interest. The funders had no role in the design of the study; in the collection, analyses, or interpretation of data; in the writing of the manuscript; or in the decision to publish the results.

\section{References}

1. Ma, W.; Lager, K.M.; Richt, J.A.; Stoffregen, W.C.; Zhou, F.; Yoon, K.J. Development of real-time polymerase chain reaction assays for rapid detection and differentiation of wild-type pseudorabies and gene-deleted vaccine viruses. J. Vet. Diagn. Investig. 2008, 20, 440-447. [CrossRef] [PubMed]

2. Pomeranz, L.E.; Reynolds, A.E.; Hengartner, C.J. Molecular biology of pseudorabies virus: Impact on neurovirology and veterinary medicine. Microbiol. Mol. Biol. Rev. 2005, 69, 462-500. [CrossRef] [PubMed]

3. Wittmann, G.; Rziha, H.-J. Aujeszky's Disease (Pseudorabies) in Pigs; Springer: New York, NY, USA, 1989. 
4. Zuckermann, F.A. Aujeszky's disease virus: Opportunities and challenges. Vet. Res. 2000, 31, 121-131. [CrossRef] [PubMed]

5. Yu, X.; Zhou, Z.; Hu, D.; Zhang, Q.; Han, T.; Li, X.; Gu, X.; Yuan, L.; Zhang, S.; Wang, B.; et al. Pathogenic pseudorabies virus, China, 2012. Emerg. Infect. Dis. 2014, 20, 102-104. [CrossRef] [PubMed]

6. Sun, Y.; Luo, Y.; Wang, C.H.; Yuan, J.; Li, N.; Song, K.; Qiu, H.J. Control of swine pseudorabies in China: Opportunities and limitations. Vet. Microbiol. 2016, 183, 119-124. [CrossRef] [PubMed]

7. An, T.Q.; Peng, J.M.; Tian, Z.J.; Zhao, H.Y.; Li, N.; Liu, Y.M.; Chen, J.Z.; Leng, C.L.; Sun, Y.; Chang, D.; et al. Pseudorabies virus variant in Bartha-K61-vaccinated pigs, China, 2012. Emerg. Infect. Dis. 2013, 19, 1749-1755. [CrossRef] [PubMed]

8. Wu, R.; Bai, C.; Sun, J.; Chang, S.; Zhang, X. Emergence of virulent pseudorabies virus infection in Northern China. J. Vet. Sci. 2013, 14, 363-365. [CrossRef] [PubMed]

9. Tong, W.; Liu, F.; Zheng, H.; Liang, C.; Zhou, Y.-J.; Jang, Y.-F.; Shan, T.-L.; Gao, F.; Li, G.-X.; Tong, G.-Z. Emergence of a Pseudorabies virus variant with increased virulence to piglets. Vet. Microbiol. 2015, 181, 236-240. [CrossRef] [PubMed]

10. Fan, J.; Zeng, X.; Zhang, G.; Wu, Q.; Niu, J.; Sun, B.; Xie, Q.; Ma, J. Molecular characterization and phylogenetic analysis of pseudorabies virus variants isolated from Guangdong Province of Southern China during 2013-2014. J. Vet. Sci. 2015, 17, 369-375. [CrossRef] [PubMed]

11. Wang, Y.; Qiao, S.; Li, X.; Xie, W.; Guo, J.; Li, Q.; Liu, X.; Hou, J.; Xu, Y.; Wang, L.; et al. Molecular epidemiology of outbreak-associated pseudorabies virus (PRV) strains in central China. Virus Genes 2015, 50, 401-409. [CrossRef] [PubMed]

12. Hu, D.; Zhang, Z.; Lv, L.; Xiao, Y.; Qu, Y.; Ma, H.; Niu, Y.; Wang, G.; Liu, S. Outbreak of variant pseudorabies virus in Bartha-K61-vaccinated piglets in central Shandong Province, China. J. Vet. Diagn. Investig. 2015, 27, 600-605. [CrossRef] [PubMed]

13. Liu, H.; Li, X.-T.; Hu, B.; Deng, X.-Y.; Zhang, L.; Lian, S.-Z.; Zhang, H.-L.; Lv, S.; Xue, X.-H.; Lu, R.-G.; et al. Outbreak of severe pseudorabies virus infection in pig-offal-fed farmed mink in Liaoning Province, China. Arch. Virol. 2017, 162, 863-866. [CrossRef] [PubMed]

14. Zhao, X.; Xu, J.; Song, X.; Jia, R.; Yin, Z.; Cheng, A.; Jia, R.; Zou, Y.; Li, L.; Yin, L.; et al. Antiviral effect of resveratrol in ducklings infected with virulent duck enteritis virus. Antivir. Res. 2016, 130, 93-100. [CrossRef] [PubMed]

15. De, L.A.; Arena, G.; Stecca, C.; Raciti, M.; Mattia, E. Resveratrol inhibits proliferation and survival of Epstein Barr virus-infected Burkitt's lymphoma cells depending on viral latency program. Mol. Cancer Res. 2011, 9 , 1346-1355.

16. Docherty, J.J.; Smith, J.S.; Fu, M.M.; Stoner, T.; Booth, T. Effect of topically applied resveratrol on cutaneous herpes simplex virus infections in hairless mice. Antivir. Res. 2004, 61, 19-26. [CrossRef] [PubMed]

17. Heredia, A.; Davis, C.; Amin, M.N.; Le, N.M.; Wainberg, M.A.; Oliveira, M.; Deeks, S.G.; Wang, L.X.; Redfield, R.R. Targeting Host Nucleotide Biosynthesis with Resveratrol Inhibits Emtricitabine (FTC)-resistant HIV-1. Aids 2014, 28, 317-323. [CrossRef] [PubMed]

18. Heredia, A.; Davis, C.E.; Reitz, M.S.; Le, N.M.; Wainberg, M.A.; Foulke, J.S.; Wang, L.X.; Redfield, R.R. Targeting of the Purine Biosynthesis Host Cell Pathway Enhances the Activity of Tenofovir Against Sensitive and Drug-Resistant HIV-1. J. Infect. Dis. 2013, 208, 2085-2094. [CrossRef] [PubMed]

19. Zang, N.; Xie, X.; Deng, Y.; Wu, S.; Wang, L.; Peng, C.; Li, S.; Ni, K.; Luo, Y.; Liu, E. Resveratrol-Mediated Gamma Interferon Reduction Prevents Airway Inflammation and Airway Hyperresponsiveness in Respiratory Syncytial Virus-Infected Immunocompromised Mice. J. Virol. 2011, 85, 13061-13068. [CrossRef] [PubMed]

20. Krishnan, V.; Zeichner, S.L. Host cell gene expression during human immunodeficiency virus type 1 latency and reactivation and effects of targeting genes that are differentially expressed in viral latency. J. Virol. 2004, 78, 9458-9473. [CrossRef] [PubMed]

21. Zhao, X.; Cui, Q.; Fu, Q.; Song, X.; Jia, R.; Yang, Y.; Zou, Y.; Li, L.; He, C.; Liang, X.; et al. Antiviral properties of resveratrol against pseudorabies virus are associated with the inhibition of IкB kinase activation. Sci. Rep. 2017, 7, 8782. [CrossRef] [PubMed]

22. Fu, Q.; Cui, Q.; Yang, Y.; Zhao, X.; Song, X.; Wang, G.; Bai, L.; Chen, S.; Tian, Y.; Zou, Y.; et al. Effect of Resveratrol Dry Suspension on Immune Function of Piglets. Evid.-Based Complement. Altern. Med. 2018, 2018. [CrossRef] [PubMed] 
23. Song, X.; Zhang, Y.; Yin, Z.; Zhao, X.; Liang, X.; He, C.; Yin, L.; Lv, C.; Zhao, L.; Ye, G. Antiviral effect of sulfated Chuanmingshen violaceum polysaccharide in chickens infected with virulent Newcastle disease virus. Virology 2015, 476, 316-322. [CrossRef] [PubMed]

24. Xu, J.; Yin, Z.; Li, L.; Cheng, A.; Jia, R.; Song, X.; Lu, H.; Dai, S.; Lv, C.; Liang, X. Inhibitory Effect of Resveratrol against Duck Enteritis Virus In Vitro. PLoS ONE 2013, 8, e65213. [CrossRef] [PubMed]

25. Dory, D.; Torché, A.M.; Béven, V.; Blanchard, P.; Loizel, C.; Cariolet, R.; Jestin, A. Effective protection of pigs against lethal Pseudorabies virus infection after a single injection of low-dose Sindbis-derived plasmids encoding PrV gB, gC and gD glycoproteins. Vaccine 2005, 23, 3483-3491. [CrossRef] [PubMed]

26. Maresch, C.; Lange, E.; Teifke, J.P.; Fuchs, W.; Klupp, B.; Müller, T.; Mettenleiter, T.C.; Vahlenkamp, T.W. Oral immunization of wild boar and domestic pigs with attenuated live vaccine protects against Pseudorabies virus infection. Vet. Microbiol. 2013, 161, 20-25. [CrossRef] [PubMed]

27. Wang, G.; Hu, Z.; Song, X.; Cui, Q.; Fu, Q.; Jia, R.; Zou, Y.; Li, L.; Yin, Z. Analgesic and Anti-Inflammatory Activities of Resveratrol through Classic Models in Mice and Rats. Evid.-Based Complement. Altern. Med. 2017, 2017. [CrossRef] [PubMed]

28. Wang, G.; Hu, Z.; Fu, Q.; Song, X.; Cui, Q.; Jia, R.; Zou, Y.; He, C.; Li, L.; Yin, Z. Resveratrol mitigates lipopolysaccharide-mediated acute inflammation in rats by inhibiting the TLR4/NF-kBp65/MAPKs signaling cascade. Sci. Rep. 2017, 7. [CrossRef] [PubMed]

29. Biron, C.A. Role of early cytokines, including alpha and beta interferons (IFN- $\alpha \backslash \beta$ ), in innate and adaptive immune responses to viral infections. Semin. Immunol. 1998, 10, 383-390. [CrossRef] [PubMed]

30. Chesler, D.A.; Reiss, C.S. The role of IFN-gamma in immune responses to viral infections of the central nervous system. Cytokine Growth Factor Rev. 2002, 13, 441-454. [CrossRef]

31. Su, H.C.; Cousens, L.P.; Fast, L.D.; Slifka, M.K.; Bungiro, R.D.; Ahmed, R.; Biron, C.A. CD4 ${ }^{+}$and CD8 ${ }^{+}$T cell interactions in IFN-gamma and IL-4 responses to viral infections: Requirements for IL-2. J. Immunol. 1998, 160, 5007-5017. [PubMed]

32. Trevejo, J.M.; Marino, M.W.; Philpott, N.; Josien, R.; Richards, E.C.; Elkon, K.B.; Falck-Pedersen, E. TNF-alpha -dependent maturation of local dendritic cells is critical for activating the adaptive immune response to virus infection. Proc. Natl. Acad. Sci. USA 2001, 98, 12162. [CrossRef] [PubMed]

33. Nguyen, K.B.; Salazar-Mather, T.P.; Dalod, M.Y.; Van Deusen, J.B.; Wei, X.Q.; Liew, F.Y.; Caligiuri, M.A.; Durbin, J.E.; Biron, C.A. Coordinated and distinct roles for IFN-alpha beta, IL-12, and IL-15 regulation of NK cell responses to viral infection. J. Immunol. 2002, 169, 4279-4287. [CrossRef] [PubMed]

34. Smith, P.M.; Wolcott, R.M.; Chervenak, R.; Jennings, S.R. Control of acute cutaneous Herpes Simplex Virus infection: T cell-ediated viral clearance is dependent upon Interferon gamma. Virology 1994, 202, 76-88. [CrossRef] [PubMed]

(C) 2018 by the authors. Licensee MDPI, Basel, Switzerland. This article is an open access article distributed under the terms and conditions of the Creative Commons Attribution (CC BY) license (http:/ / creativecommons.org/licenses/by/4.0/). 